NIST Special Publication 1900-102

\title{
Summary Report on NIST Smart Grid Testbeds and Collaborations Workshops
}

Avi Gopstein

Allen Goldstein

D.M. Anand

Paul Boynton

This publication is available free of charge from:

https://doi.org/10.6028/NIST.SP.1900-102 


\title{
NIST Special Publication 1900-102
}

\section{Summary Report on NIST Smart Grid Testbeds and Collaborations Workshops}

\author{
Avi Gopstein \\ D.M. Anand \\ Paul Boynton \\ Smart Grid and Cyber-Physical Systems Program Office \\ Engineering Laboratory \\ Allen Goldstein \\ Quantum Measurement Division \\ Physical Measurement Laboratory
}

This publication is available free of charge from: https://doi.org/10.6028/NIST/SP.1900-102

March 2021

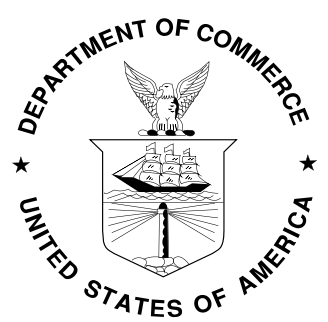

U.S. Department of Commerce Gina M. Raimondo, Secretary

National Institute of Standards and Technology James K. Olthoff, Performing the Non-Exclusive Functions and Duties of the Under Secretary of Commerce for Standards and Technology \& Director, National Institute of Standards and Technology 
Certain commercial entities, equipment, or materials may be identified in this document to describe an experimental procedure or concept adequately. Such identification is not intended to imply recommendation or endorsement by the National Institute of Standards and Technology, nor is it intended to imply that the entities, materials, or equipment are necessarily the best available for the purpose.

All registered trademarks or trademarks belong to their respective organizations.

National Institute of Standards and Technology Special Publication 1900-102 Natl. Inst. Stand. Technol. Spec. Publ. 1900-102, 43 pages (March 2021)

CODEN: NSPUE2

This publication is available free of charge from: https://doi.org/10.6028/NIST.SP.1900-102 


\section{Acknowledgements}

This report is based on the results of two Workshops on Smart Grid Testbeds and Collaborations, which were held April 11, 2019, at the University of Tennessee, Knoxville, and April 23, 2019, at the University of Vermont, Burlington. Both workshops were organized by the National Institute of Standards and Technology (NIST), an agency of the U.S. Department of Commerce.

Thanks are extended to the speakers and panelists who provided their insights on smart grid challenges, the diversity of smart grid testbed capabilities, and ways to develop a collaborative environment across these facilities through sharing information, leveraging resources, scaling experiments, and other forms of cooperation. University of Tennessee and University of Vermont provided extensive logistics and hosting support to conduct the workshops. We also appreciate the extensive contributions of the participants listed in the Appendix A; this report would not be possible without their valuable insights. Finally, thanks are extended to the Energetics team for their assistance in preparing this report.

\section{Plenary Speakers}

Avi Gopstein, NIST

Allen Goldstein, NIST

Paul Boynton, NIST

\section{Panelists}

Hantao Cui, PhD, University of Tennessee

Leon Tolbert, PhD, University of Tennessee

Ben Ollis, Power and Energy Systems Group, Oak Ridge National Laboratory

Ben Ealey, Senior Project Manager, Electric Power Research Institute

Mads R. Almassalkhi, Professor, Electrical Engineering, UVM; Co-Founder, Packetized Energy

Dr. Dan Kirk-Davidoff, Lead Research Scientist, Renewables, UL

\section{NIST Organizer}

Smart Grid and Cyber-Physical Systems (CPS) Program Office 


\section{TABLE OF CONTENTS}

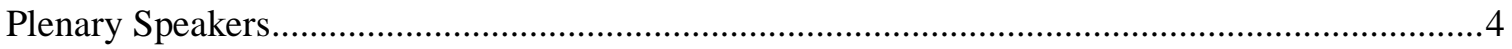

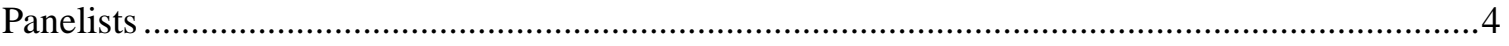

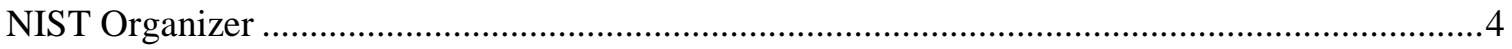

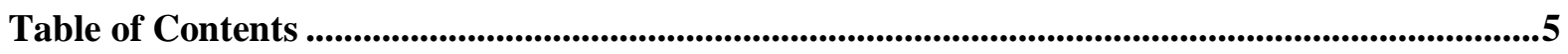

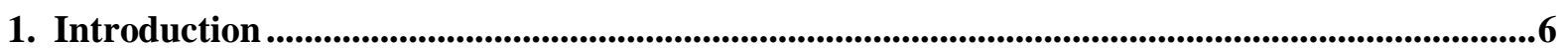

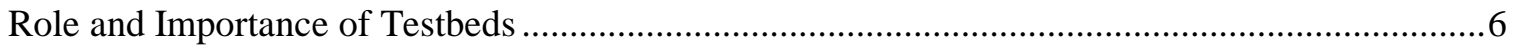

Background on the NIST Smart Grid Testbed Facility …........................................................6

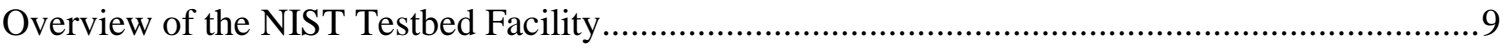

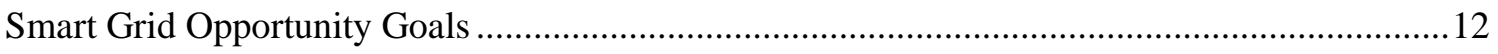

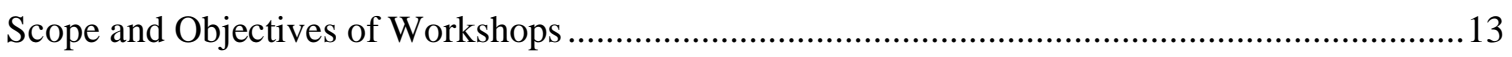

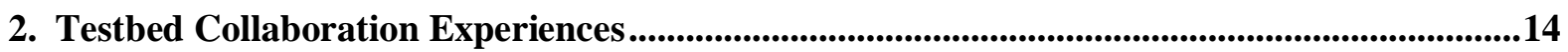

Workshop at University of Tennessee, Knoxville TN, April 11, 2019 .......................................14

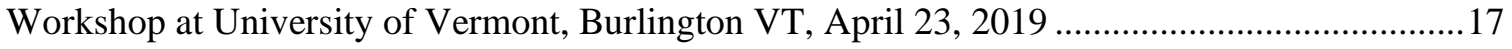

3. Stakeholder Perspectives on Testbeds and Collaboration..............................................................21

Major Challenges, Opportunities, and Gaps in Testbed Capabilities ...........................................21

Potential Roles of Collaborative Testbeds in Achieving Goals of Smart Grid Stakeholders.........28

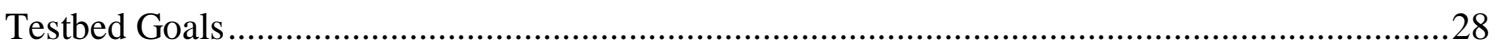

Ideal Components/Elements for Successful Testbed Collaboration............................................30

4. Next Steps.....................................................................................................................................................................33

Appendix A: Participant Lists ................................................................................................................34

Appendix B: Workshop(s) Agenda..........................................................................................................36

Appendix C: Acronyms ..............................................................................................................................................37

Addendum: Stakeholder Perspectives as recorded during breakout sessions .................................38 


\section{INTRODUCTION}

\section{Role and Importance of Testbeds}

As new energy conversion, power electronics and control technologies aim to significantly impact decades-long practices for managing the electric grid, the ability to exchange actionable information between devices and organizations has become critically important. From grid-edge intelligence to customer-owned resources, evolving grid architectures will introduce complex dynamics that span physical, economic, and information technology aspects of electrical systems.

Rapid, pervasive changes on the grid have created a growing need to test and prove new technologies before full-scale grid deployment. Greater use of renewable energy, the rising popularity of electric vehicles (EVs), increasing investment in smart home technologies, and deployment of multiple distributed energy resources (DERs) are changing how electricity is generated and distributed-with implications for stability and resilience. Utilities can now envision a system comprised of aggregated and coordinated end-user devices numbering up to tens of thousands, potentially with bi-directional power flows.

While individual technologies may work well independently, unexpected behavior can occur when they are combined into a larger system. Creating a resilient, reliable, and safe smart grid requires thorough testing and evaluation of how systems will perform and interact - prior to their deployment in practical operating environments. Realistic test environments can fulfill this requirement.

Testbeds provide a development environment without the attendant potential hazards or consequences of testing in a live production environment. Testbeds can be used to demonstrate new components or entire systems, and they may incorporate software and hardware/physical equipment as well as networking components. Testbeds are especially important for evaluating performance and identifying any potentially adverse behaviors of interacting systems before technologies are deployed.

Testbeds can support measurements of performance and other parameters and help ensure effective communication and interoperability of the equipment connected to the grid as well as other infrastructure. Testbed environments are valuable in addressing numerous measurement and standards challenges related to key grid technologies (e.g., power electronics, power metering, and energy storage).

\section{Background on the NIST Smart Grid Testbed Facility}

\section{Presented by Avi Gopstein of NIST}

The evolving smart grid uses information and communication technology to make the power grid more efficient, reliable, secure, and resilient while minimizing costly investments in new generation capacity. The Energy Independence and Security Act of 2007 (EISA) established the development of the smart grid as a national policy goal and assigned NIST the "primary responsibility to coordinate development of a framework that includes protocols and model standards for information management to achieve interoperability of smart grid devices and systems ..."

Development of this national framework for information exchange on the evolving grid is predicated on a clear understanding of the underlying physics and corresponding measurements. As the national metrology lab, NIST is well positioned to undertake the task of analyzing the required resolution and 
accuracy of system measurements which in turn will help to determine the requirements for information exchange, enabling the interoperability of devices and systems. A full understanding of the physical processes being measured can then help to identify effective control strategies and reliable methods to convey control signals. The need to understand these smart grid phenomena led to development of the NIST Smart Grid testbed.

As a first step, NIST developed a conceptual model of the grid, showing the key stakeholders or domains and how they interact. As shown in the draft model in Figure 1, the various physical interactions (electricity flows, in dashed gold lines) are clearly distinguished from communications flows (solid blue).

The scenario in Figure 2 (below) highlights important communications interfaces between and within domains of the smart grid in a scenario with larger numbers of distributed energy resources (DERs). Figure 2 is intended to illustrate that the communication infrastructure needed to support all interacting components and systems can be fairly complex.

Digitization and rapidly dropping costs are accelerating the adoption rates and diversity in DERs across domains. For example, a sharp drop in the cost of utility-scale photovoltaics (PV) is fostering rapid growth in installed PV generation capacity. ${ }^{1}$ Over eight years, the cost of sensors has dropped about $98 \%$ contributing to an increasing volume in measurement data and focus on information technology and data management across a range of domains. By 2040, most household electricity consumption is expected to flow through network-enabled devices. ${ }^{2}$

\footnotetext{
${ }^{1}$ Barbose G, Darghouth N (2019) Tracking the Sun: Pricing and Design Trends for Distributed Photovoltaic Systems in the United States-2019 Edition. (Lawrence Berkeley National Laboratory, Berkeley, CA). Available at: https://eta-publications.lbl.gov/sites/default/files/tracking the sun_2019_report.pdf

${ }^{2}$ International Energy Agency (2017) Digitalization \& Energy. (IEA, Paris, France). Available at: https://www.iea.org/publications/freepublications/publication/DigitalizationandEnergy3.pdf Figures 2.5 and 4.4 present data relevant to the claims made in this report.
} 


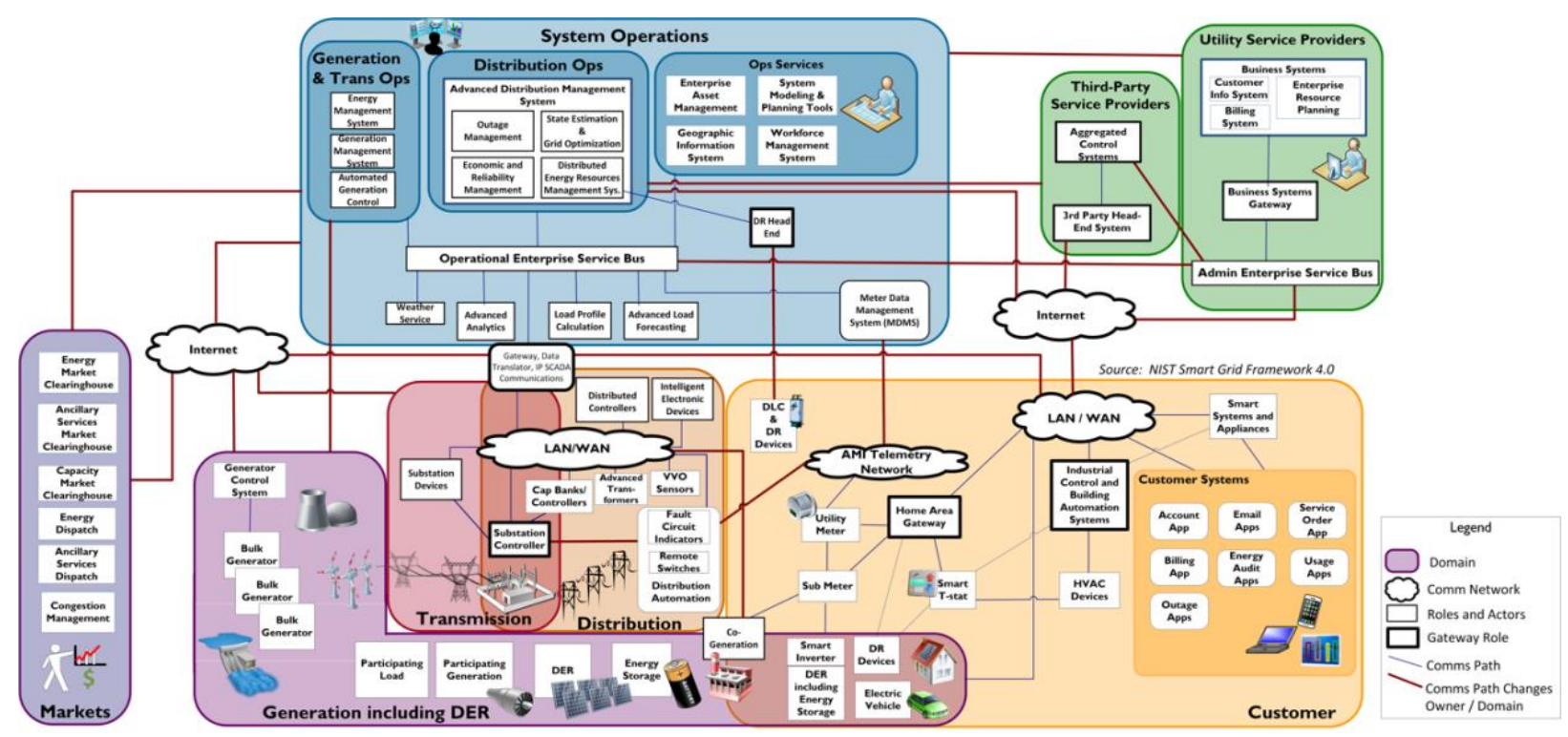

Figure 2: NIST Interoperability Model showing a High-DER Communication Pathways Scenario. The diagram highlights the diversity in data interfaces and inter-domain interactions.

The interoperability model in Figure 2, along with the other scenarios outlined in the NIST Framework and Roadmap for Smart Grid Interoperability Standards, Release $4.0^{3}$ tries to anticipate the diversity of DERs and where they are likely to fit within the smart grid. It shows the role of intelligent distribution systems and the empowerment of end users - extending beyond the residence to include new market platforms. Many customer-owned and customer-sited assets (e.g., customerowned smart inverter or electric vehicle) serve dual purposes: they serve the customer but can also serve the grid. This overlap raises an important conditionality issue for information exchange.

These new options will force system operators to consider whether information should be shared via the internet or directed by proprietary means. As tension grows between centralized and distributed control schemes, the choice of information pathways and control strategies must carefully consider the potential grid impacts of smart thermostats, sensors, and distributed resources. For example, if too many informed consumers or pre-programmed devices react strongly to utility price signals, might their reaction over-correct the earlier imbalance and create an opposite yet equally undesirable situation?

Recognizing that no single grid architecture is likely to dominate, NIST has developed multiple scenarios (e.g., micro grids and hybrid systems) that allow us to examine interoperability requirements from different perspectives. In these scenarios diverse control algorithms and communication interfaces are required, and devices at the edge of the system increasingly rely on a shared infrastructure. Identifying the diverse options for communications and control may help to identify which interfaces could be most useful to normalize. The NIST smart grid testbed facility is intended to help test, measure and evaluate the aforementioned control algorithms and communication interfaces in order to complement the insights and ideas in the Framework and Roadmap for Smart Grid Interoperability.

NIST has proposed four main use cases for its testbed:

\footnotetext{
${ }^{3}$ Gopstein AM, Nguyen CT, O’Fallon CM, Hastings NE, Wollman DA (2021) NIST Framework and Roadmap for Smart Grid Interoperability Standards, Release 4.0. (National Institute of Standards and Technology, Gaithersburg, MD), NIST SP 1108r4. https://doi.org/10.6028/NIST.SP.1108r4
} 


\section{Characterize required observability}

a. Understand the harmonics, resource variabilities, and non-linearities in the system and how they propagate between DERs and the grid; understand impacts on transducer design and metrology to enhance our ability to observe and understand what is happening on the system.

b. Conduct sensitivity analyses for all components of observability to guide frequency and clarify the uncertainty of measurements (particularly waveforms not previously available).

\section{Scaling stability (bulk/distribution)}

a. Look at the effects of device interaction on power quality at the customer level and how those effects translate to the distribution system.

\section{Hardware-in-the-loop R\&D}

a. Accurately emulate the grid interfaces and power electronics to help utilities feel confident that a technology will work on their system.

i. Working now with the Department of Energy's national laboratories that use hardware-in-the-loop validation to identify specific emulation challenges.

\section{Advance NIST expertise}

a. Develop knowledge and insights to support standards development efforts.

b. Standards development organizations and working groups actively seek NIST participation because of the expert knowledge gained from NIST research and testbeds.

c. They also recognize that NIST has no vested interests in any particular technology; it is a trusted broker in providing unbiased data.

NIST encourages stakeholders to identify new use cases for testbeds likely to provide the greatest value.

\section{Overview of the NIST Testbed Facility}

Presented by Allen Goldstein, NIST

NIST performs vital research supporting the nation's electrical industry and infrastructure. Drawing on its scientific, technical, and engineering expertise, NIST conducts research related to smart grid metrology. As part of its technical efforts to support this mission, NIST has established an interactive Smart Grid Interoperability Testbed Facility (SGTF). The testbed allows NIST scientists, engineers, and researchers from partner organizations to explore smart grid interoperability among many different components in many different situations. ${ }^{4}$

Using the SGTF, NIST researchers are able to address the needs of the evolving smart grid community by performing research needed to inform the development of smart grid standards. NIST is a leader in contributing technical expertise to standards development, evaluating test protocols, and supporting the development of meaningful requirements for interoperability. NIST testbed research helps to improve the understanding of distribution system dynamics at the grid edge and to translate that understanding into observable requirements.

\footnotetext{
${ }^{4}$ https://www.nist.gov/el/smart-grid/smart-grid-interoperability-testbed
} 
The unique strength of the NIST Testbed derives from the NIST mission, mandate, expertise, and focus as an unbiased, peer-reviewed, and fully funded federal research agency. Although the testbed incorporates some prototype hardware not yet available in the market, much of its hardware is commercially available.

The SGTF is a unique set of interconnected modules that in combination provide grid emulation capabilities across multiple laboratory spaces linked by an electrical and communications bus. The shared bus is designed to enable a broad range of experiments in interoperability, with a focus on precise measurement. Each of the modules are briefly described below.
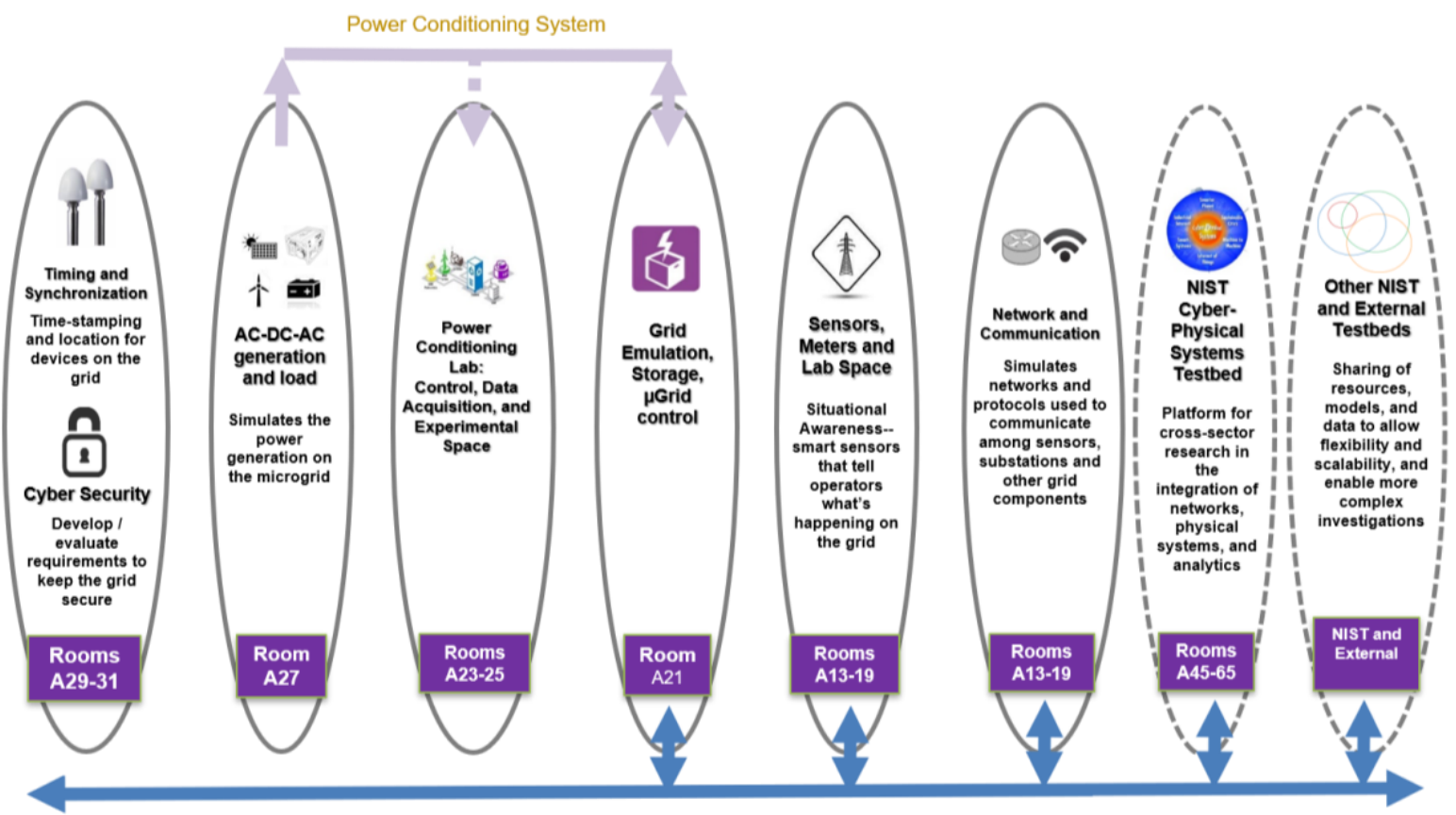

Figure 3: NIST's Smart Grid Testbed facility is comprised of a set of interconnected modules.

The Timing \& Synchronization (Synchrometrology) Lab uses multiple external resources for accurate time signals and measurements. The sources include a Stratum 1 Cesium clock array synchronized to UTC(NIST) via common view time transfer, a local Stratum 2 clock synchronized to within a nanosecond of the Cesium clock array using the White Rabbit time transfer protocol, ${ }^{5}$ and lastly multiple calibrated GPS timing receivers operating as alternate Stratum 1 clocks.

Correct time and timing are foundational elements in enabling the communication and orchestration of technologies for accurate and optimal wide area monitoring, protection, and control in the power industry. The lab is designed to research and test components requiring precision time stamps essential for smart grid applications, enabling operators to effectively maintain grid balance amid rapidly changing energy inputs and loads.

The Power Conditioning System (PCS) is comprised of power conversion and control equipment located in two firewalled rooms (Rooms A27 and A21 in Figure 3) with a laboratory space in between. The PCS area has access to three independent three-phase electrical buses. It enables some microgrid research and supports experiments in other areas of the testbed by generating a wide range

${ }^{5}$ Dierikx, Erik F., et al. "White Rabbit precision time protocol on long-distance fiber links." IEEE Transactions on Ultrasonics, Ferroelectrics, and Frequency Control 63.7 (2016): 945-952. 
of waveforms reflecting real world power system signals. Multi-tone, modulated waveforms can be generated under controlled conditions to test equipment response.

PCS refers to the general class of devices that use power electronics technologies to convert electric power from one form to another; for example, converting between direct current (DC) and alternating current (AC). Many loads and sources on the power grid today are already interfaced through PCSs to provide the type of electricity needed by the load, and they also provide valuable grid interface characteristics. Although only a fraction of the power generation on the grid today is PCS based, the penetration levels of PCS-based generation and storage are increasing very rapidly due to the addition of renewable/clean energy sources that produce DC (e.g., photovoltaic and fuel cell) or variable AC (e.g., wind turbines) electricity.

Early standards and implementations for grid integration of distributed generation and storage (Distributed Energy Resources or DER) were intended for low penetration levels and were not originally designed to ensure grid stability and safety at high-penetration levels. A new approach calls for PCS to provide an operational interface for DER that better meets these needs, enabling higher penetration levels and values for DER devices.

This new approach is paving the way for future PCS-based grid architectures using microgrids to enable very high penetration levels of DER, improved grid resiliency, and rapid grid restoration and recovery. ${ }^{6}$

The AC/DC and A/C Generation Lab has grid connections to enable simulated power generation and load. Regenerative grid emulators can be tied together, and each device can be tied into the threephase buses. In close proximity are the relays, microcontroller devices, and micro grid controllers under test as well as some grid emulation, battery storage, and micro grid controllers. All these components are sometimes collectively referred to as a distributed energy resource management system (DERMS).

Electricity meters today face unprecedented challenges, from distorted waveforms on the grid, bidirectional metering for renewables, and the use of meters as distribution grid sensors for monitoring and control. ${ }^{7}$ The Sensor $\&$ Metering Lab is designed to verify meter accuracy under real-world harmonic distortion of voltage and current, temperature fluctuation and electromagnetic interference. Accuracy testing was first designed to measure $0.2 \%$ error tolerance and then upgraded to an accuracy of $0.05 \%$ to support the best commercially available meters anticipated in the near future. The lab is also equipped to conduct distorted and bi-directional flow testing on smart meters.

The Network and Communication Lab provides a research testbed capable of evaluating and demonstrating the performance of various grid and communication network technologies in real-time and under real world conditions. The testbed is specifically designed to support smart grid applications with significant dependence on real-time communication. It is based on an emulation platform that allows a large number of virtual nodes to be created to communicate in a centralized or distributed manner. ${ }^{8}$

The Cyber-Physical Systems Testbed is a specially designed private network linking all rooms and components across the facility and provides a high level of integration for instrument control and data acquisition.

\footnotetext{
${ }^{6}$ www.nist.gov/programs-projects/power-conditioning-systems-renewables-storage-and-microgrids

${ }^{7}$ wwW.nist.gov/programs-projects/advanced-metering-smart-distribution-grids

${ }^{8}$ www.nist.gov/programs-projects/combined-grid-communication-testbed-implementation-real-time$\underline{\text { measurement }}$
} 
Microgrid Test System: A pair of inverters $(10 \mathrm{~kW}$ and $30 \mathrm{~kW})$ can be used to test various microgrid controllers - both commercially available brands and those developed in house.

NIST's Gaithersburg site also contains a Net Zero Energy residential facility ${ }^{9}$ with a closely monitored $30 \mathrm{~kW}$ roof solar array as well as another $5 \mathrm{MW}$ solar array; the data from these systems is available to the public and shares data with the SGTF in real time.

In summary, the NIST testbed is designed as a platform to measure and characterize smart grid operations and communications to advance measurement science as the foundation for many grid modernization priorities. Based on stakeholder input, NIST is working to expand understanding of grid operations (beyond the steady-state models) to include security, latency, and uncertainty.

\section{Smart Grid Opportunity Goals}

EISA Title XIII [Section 1301] makes it U.S. policy to support grid modernization to achieve the following smart grid objectives:

1. Increased use of digital information and controls technology to improve reliability, security, and efficiency of the electric grid.

2. Dynamic optimization of grid operations and resources, with full cybersecurity.

3. Deployment and integration of distributed resources and generation, including renewable resources.

4. Development and incorporation of demand response, demand-side resources, and energy-efficiency resources.

5. Deployment of "smart" technologies (real-time, automated, interactive technologies that optimize the physical operation of appliances and consumer devices) for metering, communications concerning grid operations and status, and distribution automation.

6. Integration of "smart" appliances and consumer devices.

7. Deployment and integration of advanced electricity storage and peak-shaving technologies, including plug-in electric and hybrid electric vehicles, and thermal-storage air conditioning.

8. Provision to consumers of timely information and control options.

9. Development of standards for communication and interoperability of appliances and equipment connected to the electric grid, including the infrastructure serving the grid.

10. Identification and lowering of unreasonable or unnecessary barriers to adoption of smart grid technologies, practices, and services.

Smart grid technology enables a diverse energy mix, increased participation by customers, and resilient and reliable grid operations.

https://www.energy.gov/sites/prod/files/oeprod/DocumentsandMedia/EISA Title XIII S mart_Grid.pdf

\footnotetext{
${ }^{9}$ https://www.nist.gov/el/net-zero-energy-residential-test-facility
} 


\title{
Scope and Objectives of Workshops
}

\author{
Presented by Paul Boynton
}

Achieving industry consensus on interoperability standards for the evolving and future smart grid is greatly complicated by the rapid expansion and growing diversity of distributed resources, consumerowned energy devices, energy storage options, and power control equipment. Greater collaboration among all stakeholders can help to accelerate technology progress, technical understanding, and broad consensus across sectors.

NIST takes an inclusive view of collaboration as a means to bring together diverse perspectives, areas of expertise, resources, equipment, and technology on a variety of scales. Collaborative efforts tend to achieve the best results when expert knowledge overlaps in the area of interest.

“...science is most effective when researchers with expert knowledge in different areas collaborate on a project of overlapping interest. The overlap allows for common ground, while the respective areas of expertise [and other resources] cover a greater "surface area" of the possible knowledge brought to bear on a specific question."

- Donald T. Campbell, "Ethnocentrism of Disciplines and the Fish-Scale Model of Omniscience.” (1969)

The collective intelligence and cross-fertilization of ideas can facilitate innovative solutions that work for all parties. Another benefit is wide buy-in, accelerating market acceptance and uptake of the resulting solutions, technologies, and standards. NIST provides unbiased technical data and evaluations, enabling productive collaborative efforts.

In April 2019, NIST held regional workshops in Tennessee and Vermont to explore the diverse capabilities of smart grid testbeds and identify ways to enhance a collaborative environment across facilities to share information, leverage resources, scale experiments, and accelerate progress. Workshops were co-hosted by two local universities with unique testbed capabilities:

- University of Tennessee, Knoxville, TN, April 11, 2019

- University of Vermont, Burlington, VT, April 23, 2019

These public workshops offered sessions to explain and gain feedback on the NIST testbed project and highlight related activities by academic and industry experts, including the development and operation of other testbed facilities. Workshop participants were given the opportunity to present their views on testbed needs and challenges during facilitated discussions. A complete list of workshop participants is provided in Appendix A. A typical workshop agenda is included as Appendix B. Appendix C provides an Acronyms List, while Appendix D offers links to useful webpages.

This report represents a summary of the discussions that took place during the workshops. It includes highlights of speaker presentations as well as the major ideas and concepts generated during facilitated sessions. Ideas that are specific to certain regions are indicated where appropriate.

Note that the results presented here do not reflect those of the entire smart grid community; they represent a snapshot of perspectives based on those who attended and participated in discussions at the two workshops. Invited attendees included a range of individuals with diverse backgrounds in grid modernization as well as testbed operation. 


\section{Testbed Collaboration Experiences}

The following summaries provide highlights of experiences with testbeds and collaborations presented by several panelists. ${ }^{10}$

\section{Workshop at University of Tennessee, Knoxville TN, April 11, 2019}

\section{Hantao Cui, University of Tennessee (UTK)}

UTK currently operates a large-scale testbed for grid simulation. The testbed focuses on the monitoring and control of power electronic devices to strengthen fundamental knowledge and develop enabling technologies.

For integration purposes, the testbed includes both a hardware testbed and large-scale testbed. In the large-scale testbed, a real-time grid platform is being developed to continuously simulate the grid during disturbances. While traditional simulators look only at physical devices, the UTK testbed investigates the communications layer, including network-based communications devices and control implementation. An interactive web interface is needed so that users can see and interpret the results. Initially, our researchers modeled the full North American grid and reduced the dynamic models to a practical size for software simulation. About 20\% total nameplate generation was modeled as renewable generation to represent the grid of the future. Our researchers are now developing an open source simulator for the power system and investigating wide-area

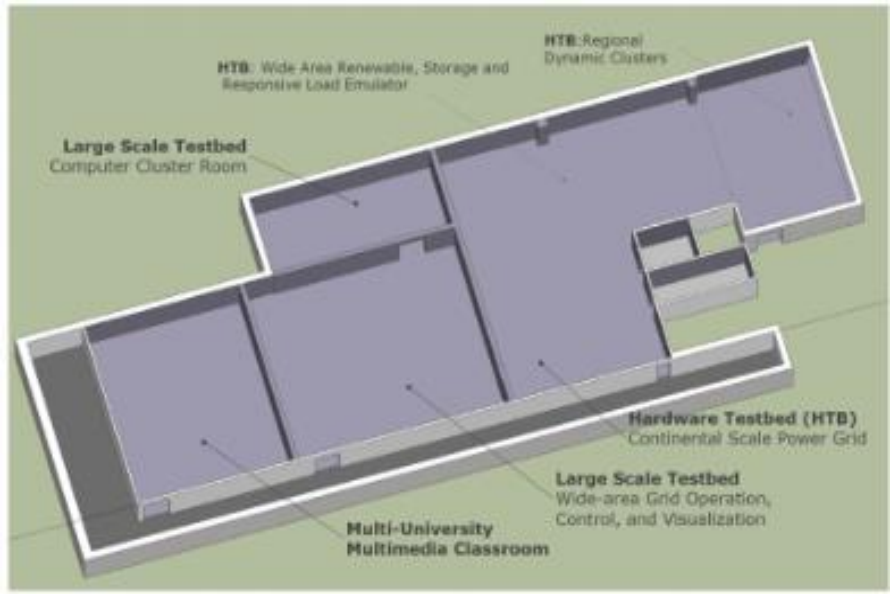

Figure 4: University of Tennessee Power Systems Simulation Laboratory. Photo Credit: CURENT, UTK. Used with permission. measurement and controls. The third-generation testbed will add more renewable energy capacity, energy storage, and a communications network emulator.

In the large-scale testbed, modules are glued together through a communication network. A closed loop emulator incorporates an open source power system simulator (called ANDES) to allow data streaming. This setup allows researchers to simulate the measurements in the testbed by looking at the raw data from the simulator. A unique characteristic of the testbed is its use of an in-house-developed visualization platform (called LTBWeb) that lets the user change parameters and turn control algorithms on or off, bringing power and communication together. On the cybersecurity side, the testbed can simulate a false data attack on a PMU based monitoring system. In this attack, a sophisticated attacker records PMU data indicative of a fault and replays it in place of actual measurements even when the actual power system is stable.

\section{Leon Tolbert, University of Tennessee (UTK)}

\footnotetext{
${ }^{10}$ These keynote descriptions are not a transcript of the remarks delivered; instead, they are summaries providing presentation highlights.
} 
The hardware testbed at UTK uses power electronic converters to emulate the electric grid. Researchers are emulating the bulk power grids overseen by the Northeast Power Coordinating Council and Western Electricity Coordinating Council (WECC), focusing on the high penetration of renewables and use of multi-terminal, high-voltage, direct current (HVDC) to move power over long distances. The emulation structure is such that converters can be programmed to emulate generators or loads with different characteristics. When taking measurements, it can be difficult to tell whether you are looking at a real load/generator or one emulated with power electronics. UTK has developed several emulators, including ones for wind, solar, three phase loads, energy storage, transmission line emulators with faults, synchronous generators, and a real-time interface to enable real-time digital power system simulation.

More recently, our researchers have investigated the North American grid, WECC, EI, and ERCOT. With more renewables, the idea is to pass energy across interconnections and have hardware emulators for those interconnections. They also use the testbed for microgrid emulation, in partnership with EPB Chattanooga- a regional electric utility. EPB has the interrupters, and UTK is testing the controller before it is implemented on their system. Testing involves reconfigurable control, visualization, and interoperability among multiple simultaneous control functions. The test is designed to answer questions such as: How do multiple systems affect each other when running at the same time (e.g., state estimation, event estimation, grid operation, etc.)?

UTK continues to examine and refine emulation and controller models, integrate software with the hardware testbed, and explore cybersecurity events.

\section{Ben Ollis, Oak Ridge National Laboratory (ORNL)}

Testbeds at ORNL focuses on microgrid research and capabilities, from transmission and distribution modeling to controls. Individual testbeds can be integrated to expand capabilities. Current testbeds include CSESMIC (pronounced seismic), DECC microgrid, SI-GRID (ultra-low voltage microgrid emulation platform), and HIL platforms (RTDS, Opal-RT). The testbed was built as part of a DOE project to look at microgrid controls (distributed and centralized controls). The framework and architecture support advanced power system functions and offer additional layers for forecasting, visualization, and communications. The built intelligence nodes can be placed onto devices; for example, if a controller indicates, 'I want to activate my energy storage,' it can initiate that process.

Our researchers worked on a simulation for a moderate residential neighborhood, integrating VOLTTRON and pseudo transactive controls. In the simulation, dynamic prices are issued, and a load profile is returned from the home, saying what the home is willing to do in terms of load profile changes. FPGA-based controllers have been used to control the switching behavior of the inverters (flexible parameters) and to communicate directly to the inverters through the microgrid controller. The testbed provides a flexible method to test in simulation before moving up to a full-power system (i.e., using different types of controllers, communication latencies, etc.). Commercial inverters do not have the same capabilities as the testbed; the testbed uses an inverter that interfaces with testbed controllers and is fully controllable. The testbed can simulate both a direct grid connection as well as an islanded circuit. The testbed can also fully synchronize islanded circuits. The testbed is unique, in that it represents a step between hardware emulation and full power hardware setup for use in investigating both faults and recovery. 


\section{Ben Ealey, Electric Power Research Institute (EPRI)}

EPRI is an independent, non-profit research center operating for the benefit of the public. Members include utilities, businesses, governments, and international utilities. EPRI's Distributed Energy Resource Management System (DERMS) testbed is helping stakeholders understand how to communicate and interact with many different devices. EPRI is interested in translation capabilitiestalking to other devices that use different protocols and are located at different places on the network.

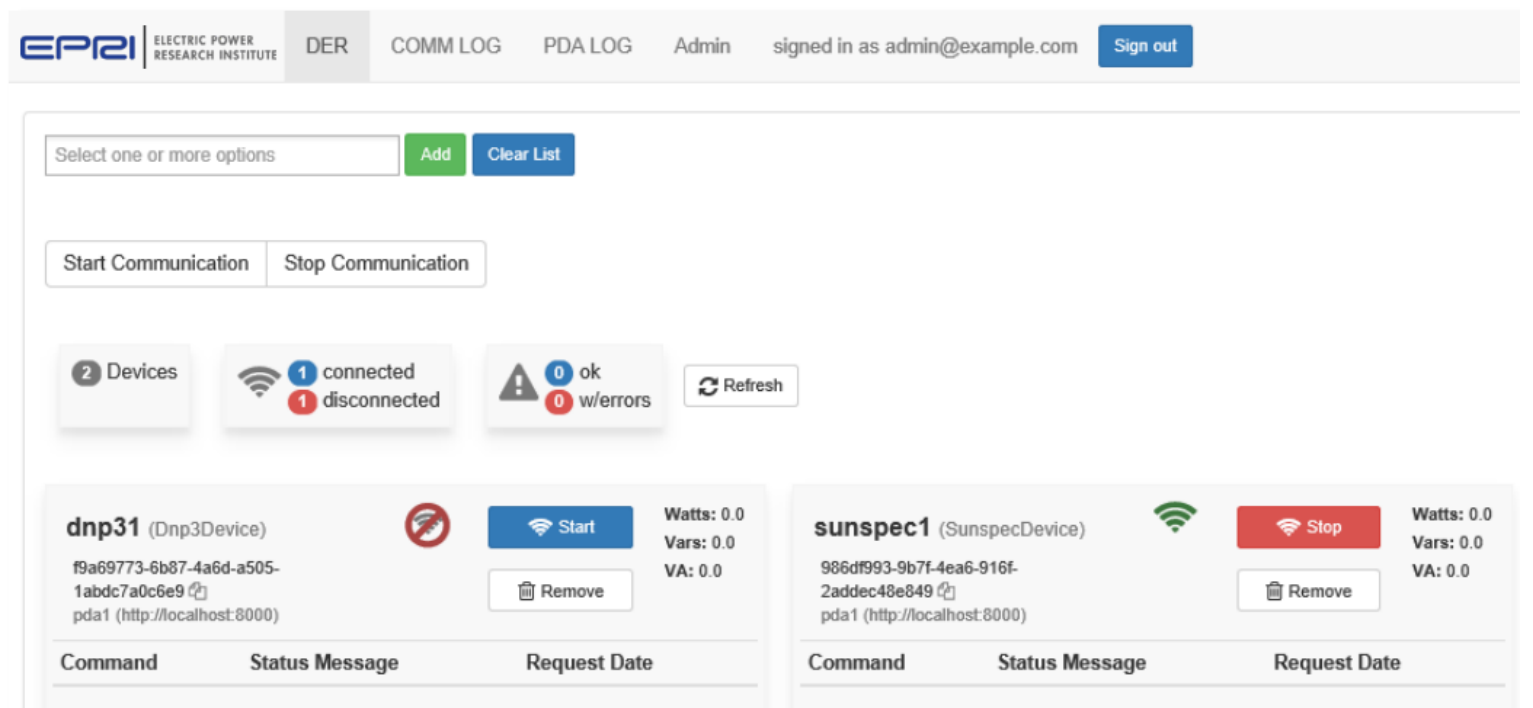

Figure 6: A screenshot showing the OpenDERMS user interface. CEPRI. Used with permission. Original image available at www.epri.com/\#/pages/product/000000003002013623

The testbed is used to evaluate the impact of DERMS on the distribution grid. The testbed can emulate thousands of different types of DER. With these virtualized devices, the testbed can then create models of how the devices will interact in different configurations and grid scenarios in the field. These models enable the development of new control strategies based on the DER in use today. Vendors design their technology for a range of operating conditions and often create proprietary control schemes for their devices. By plugging these devices into the emulator, the testbed is able to test actual hardware in different circumstances to better understand its behavior.

Enterprise DERMS can be expensive to buy and complex to operate. EPRI created an open-source reference implementation of a DERMS called OpenDERMS, which is available by request at no cost. It includes tools to receive reports from an aggregator and model different grid component topologies (e.g., nesting resources like a microgrid or an aggregator). OpenDERMS also includes models to emulate real devices and ensure that the modeled behavior mimics real-life behavior.

EPRI has cataloged its resources in the free report, "EPRI's Distributed Energy Resources Integration Toolkit," available on the EPRI website. ${ }^{11}$ These tools are highly scalable and can be used to evaluate other systems. These products are currently used in research to better understand the grid. In conjunction with the U.S. Department of Energy's National Renewable Energy Laboratory (NREL) and Sandia National Laboratory (SNL), EPRI provides a database and some device simulators to support simulation of grid scenarios. Some utilities are using the toolkit to understand more about the current state of DERMS, generally. EPRI is also working with vendors to integrate these products into their systems.

${ }^{11}$ https://www.epri.com/research/products/000000003002013623 
As EPRI continues to develop the testbed and create a real-world emulation of state-of-the art capabilities, EPRI will launch a user's group testbed, providing access to these tools and collecting user feedback.

\section{Workshop at University of Vermont, Burlington VT, April 23, 2019 Mads R. Almassalkhi, University of Vermont (UVM)}

UVM is relatively small and has a limited amount of hardware in the loop testbed capabilities-yet is able to do important power systems research. UVM is fortunate to have bright and enthusiastic grad students and faculty members as well as excellent partners, including the Vermont Electric Power Company (VELCO), Green Mountain Power (GMP), ARPA-E, ConEdison, Orange and Rockland, DOE/Office of Energy Efficiency and Renewable Energy (EERE), the National Science Foundation (NSF), and NIST. Over the past two years, some grad students led by Adil Khurram, set up a cyberphysical platform for running technical and practical tests on the algorithms that are developed in the lab. (The lab was named The Energy and Systems Lab (TESLA) - prior to the existence of any company with the same name).

The UVM Packetized Energy Management (PEM) project, funded by ARPA-E, leverages two nationwide trends: the state-led push toward clean, renewable energy and greater connectivity among resources. The goal is to create algorithms that turn these connected components and energy resources into virtual batteries or virtual power plants that can smooth out the transition to a renewable future.

Vermont generates a large amount of clean energy, particularly solar, but the balance can change dramatically from day to day. Instead of using rapid-ramp-up peaker plants, the utility is exploring ways to coordinate connected loads and tap thousands of hot water heaters and other devices for cleaner and more affordable energy. This process naturally requires robust control algorithms. Ultimately, the system should allow millions of small end-use devices to cooperatively balance energy supply and demand in real time without jeopardizing the reliability of the grid or the quality of service to consumers.

UVM is now using its recently constructed test platform to evaluate the algorithms it has developed for coordinating connected loads and making use of the relatively free energy in people's water heaters. The research uses the testbed platform to aggregate loads, figure out how they behave in aggregate, and how to control them in a way that will also keep utility customers happy (e.g., no cold showers). Many Vermonters are buying backup batteries, but cyber-physical schemes for load coordination can potentially be cheaper than lithium-ion batteries.

The project focuses on the use of packetized energy management, which involves two core principles:

- Take large, bulky demand and chop it into small packets of demand

- Through randomization, make sure that all packets are not consumed at once (during peaks).

The algorithm is basically a control loop: a system with bi-directional communications between the devices and the aggregator. The interactions are modeled on a computer to simulate impacts on the grid. The idea is to get the devices to behave a certain way in aggregate to enable control. By controlling demand, it can become a virtual power plant or virtual battery, avoiding peaks in electricity rates. 
The team simulated 500 to 5,000 water heaters in the lab and tracked the power reference signal. The tests showed that MW resources fluctuated by several megawatts, but water heater temperatures fluctuated little, so utility customers could stay comfortable. While individual demand may change, the aggregate demand stays relatively steady, providing the grid with a flexible resource.

\section{Battery designed to power 1000 homes for four hours $( \pm 1 \mathrm{MW}$, $4 \mathrm{MWh})$}

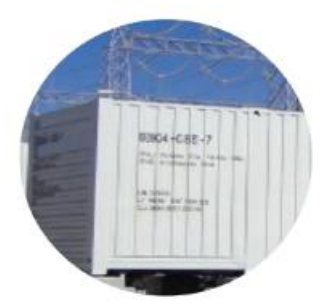

\begin{tabular}{|r|r|r|}
\hline & per $\mathrm{kWh}$ & \multicolumn{1}{c|}{ Total } \\
\hline Upfront cost & $\$ 450$ & $\$ 1,800,000$ \\
\hline Ongoing O\&M cost & $\$ 5 / \mathrm{yr}$ & $\$ 20,000 / \mathrm{yr}$ \\
\hline Customer dividend & $\$ 0$ & $\$ 0$ \\
\hline Present value cost & $\$ 481$ & $\$ 1,922,891$ \\
\hline
\end{tabular}

\section{Equivalent Packetized Virtual Battery} (2000 devices, $\pm 1 \mathrm{MW}, 4 \mathrm{MWh}$ )

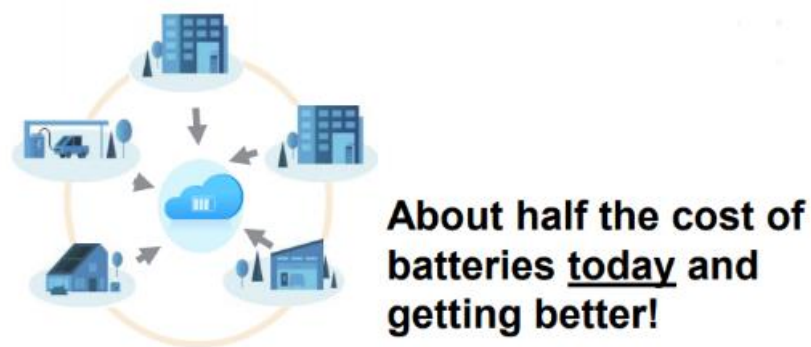

\begin{tabular}{|l|r|r|r|} 
& Per device & per kWh & \multicolumn{1}{c|}{ Total } \\
\hline Upfront cost & $\$ 200$ & $\$ 100$ & $\$ 400,000$ \\
\hline Software & $\$ 30 / y r$ & $\$ 15 / y r$ & $\$ 60,000 / y r$ \\
\hline Customer dividend & $\$ 30 / y r$ & $\$ 15 / y r$ & $\$ 60,000 / y r$ \\
\hline Present value cost & & $\$ 284$ & $\$ 1,137,348$ \\
\hline
\end{tabular}

Figure 7: Costly backup batteries could potentially give way to virtual batteries using packetized energy. (CPacketized Energy. Used with permission. Available online: https://arpae.energy.gov/sites/default/files/UVM\%20NODES\%20Annual\%20Meeting\%202019.pdf

The UVM team also applied their algorithm on 10 (approximately $10 \mathrm{kWh}$ each) batteries. The simulation results showed that with a larger number of batteries $(1,000)$, the performance of the coordination and tracking algorithm became improved.

Working with Vermont Electric Co-Op, the team tested 60 water heaters in field, and the algorithm appears to be working as expected. This small, simple demonstration does not adequately test communications limits; the scheme needs to be tested at a scale where control inputs could alter the grid state or dispatch set points. Questions remain on flexibility and uncertainty of the control algorithm when used on a large-scale deployment. The team plans to gradually scale up to a larger number of devices of various types, such as electric vehicles, back-up batteries, and HVAC systems. Along the way, the research will explore ways to optimize the system. In addition, researchers will try to identify limits or constraints on operations in distribution circuits, such as issues with voltage meters or protection systems.

Prior to integrating the algorithms on the grid, the team needs to conduct more simulations with realworld data. Unfortunately, utility data comes in different formats, so the team has built an interface funnel for the data and modeled the topology of the circuit to build time-series simulations. This work is being conducted in partnership with Green Mountain Power, Sandia National Laboratory, and Orange and Rockland to examine a range of scenarios.

UVM wants to use the developed hardware, software, and algorithms to help utilities answer questions about options for managing loads when they have a large share of intermittent resources. The team is applying filters to the available data and using cyber coupling to estimate the state of charge of every device at any given point in time; the limits are still being tested. UVM received a 
two-year extension on the research, so the team is moving toward faster time scales and larger service territories, while keeping communications at a scalable level.

Packetized energy control schemes have the potential to enable real-time coordination between distributed generation, such as rooftop and community solar assets and bulk power generation, while proactively shaping electric load. This could alleviate periods of costly peak demand, reduce wasted energy, and increase renewables penetration on the grid.

\section{Dan Kirk-Davidoff, Underwriters Laboratories (UL)}

Utilities, grid operators, solar power plant owners, and other stakeholders increasingly need to know when, where, and how much solar power will be produced at specific locations in the United States. More accurate solar forecasting will allow power system operators to integrate more solar energy into the electricity grid and ensure the economic and reliable delivery of renewable energy to American families and businesses.

The Renewables Division at UL conducts solar and wind forecasting services for utilities, regional transmission organizations (RTOs), ISOs, and other organizations. In addition, UL conducts scientific research in forecasting for the New York State Energy Research and Development Authority (NYSERDA), the California Energy Commission (CEC), and the Department of Energy.

To meet the needs of utilities with increased distributed energy resources, the time resolutions for forecasting are continuously shrinking. At one time, daily forecasts were perfectly adequate. As more renewable technologies came down in price and were deployed more widely, the update interval became just four hours. Time intervals between updates have now shrunk to an hour at most, and they are rapidly moving toward 15 minutes, 5 minutes, or less.

The need for more frequent forecasts of solar and wind availability inherently raises issues of tradeoffs in accuracy. Forecasters now must weigh the accuracy of the forecast against a range of transactional data for different weather characteristics.

A better understanding of the variability of weather and the error/uncertainty of forecasts will help grid operators and others recognize weather's multi-faceted impact on the grid. Typically, wind resources increase overnight, whereas solar power is naturally limited to daytime. Solar availability is highly variable from day to day, depending upon the weather characteristics each day.

With the massive increase in photovoltaic (PV) panels in California, solar capacity can now exceed inverter capacity, so solar energy can be maxed out during peak sunshine hours (inverter clipping), flattening the top of the solar generation curve. Inverters are most efficient at full capacity, so additional inverters are not typically an economic solution. At the same time, many solar installations at the front end of the recent boom included controllers that continuously aim the panels toward the sun. These controllers maximize power from the rays but generally lead to very steep increases in solar generation at the beginning and end of each day, aggravating system imbalances.

It has become increasingly important to really understand the error characteristics of wind, solar, and load forecasting in a consistent way. The sources of error in each of these forecasts are physically a bit distinct.

Forecasters now have access to a wide range of data sources, including new satellite data and output from global, national, and regional models of varying resolution. Data may come from the U.S. Weather Service or foreign weather services; some are free while others must be purchased. Forecasts of temperature, wind, and solar radiation at the surface all exhibit different patterns of variability. Temperature patterns tend to be quite smooth but vary from day to day, affecting the energy demands to be met. Wind is more variable as it often changes strength frequently within a relatively short span of time; it is tends to be fairly consistent over geographic space but reacts to changes in terrain. Solar 
radiation is smooth in the absence of clouds and changes sharply at the edges of cloud cover. The challenge is to reflect these variabilities and associated levels of uncertainty in computed forecasts.

The newest generation of satellites provides much higher resolution (approximately 500 meters) and significantly improved temporal resolution. Infrared imagery reveals the vertical layers in the clouds and other details that enrich the atmospheric science. The raw satellite images have to be converted to radiance, which involves some physics to account for a whole range of variables in the atmosphere (e.g., humidity, pollution, etc.), the angle of the sun and diverse characteristics of the technologies involved. These are then interpolated to arrive at the forecast.

The wealth of data poses difficult issues and exciting possibilities. Conveying the level of forecast uncertainty as well as the uncertainty of uncertainty is a big challenge. Uncertainties associated with each forecast need to be presented in consistent form so that users can easily understand the reliability of the error bounds. By better understanding what the utilities and grid operators are trying to achieve or avoid could help forecasters maximize the usefulness of the probabilistic forecasts they generate.

UL is trying to make solar forecasts more useful at the level of distribution grids (five minutes to one minute), but more information is needed from clients who can really make use of forecasts at that resolution. Potential partners in the inverter world may have the fine-grained data. Learning more will help forecasters figure out how to better balance the quality and speed requirements. 


\section{Stakeholder Perspectives on Testbeds and COLLABORATION}

In accordance with the EISA 2007, NIST regularly engages with key stakeholders to learn about pressing smart grid interoperability issues, challenges, and approaches. As power sensing, actuation, and control continue migrating from power plants toward the grid edge, NIST invites suggestions and feedback from the growing smart grid community (including utilities, system operators, regional networks, university researchers, national laboratories, vendors, manufacturers, and end users) to inform the development of potential solutions. NIST is similarly interested in exploring the diverse range of capabilities in existing smart grid testbeds and strategies to enhance a collaborative environment. During parallel breakout sessions, the participants in both workshops were asked to respond to the following questions:

1. What are the current major challenges, opportunities, and gaps in testbed capabilities?

2. How can collaborative testbeds help achieve your organization's goals for the smart grid?

3. What are the ideal components and elements for successful testbed collaborations?

\section{Major Challenges, Opportunities, and Gaps in Testbed Capabilities}

Participants attending both workshops discussed a variety of technical challenges that smart grid testbeds may help to solve, diverse access issues affecting the use of testbeds, and some opportunities that testbeds could harness to accelerate the development of potential solutions.

\section{Challenges}

\section{Technical}

"Smart grid" technologies are expanding rapidly. At the core, they encompass two-way communication technologies, control systems, and computer processing. Advanced sensors known as Phasor Measurement Units (PMUs) allow operators to assess grid stability. Advanced energy meters give consumers information about their usage to help them improve energy efficiency meters also automatically report outages. Relays sense faults in the substation and automatically aid recovery. Automated feeder switches re-route power around problems, and batteries store excess energy for later grid use to meet customer demand.12 This positive transformation introduces an array of technical challenges.

\footnotetext{
${ }^{12}$ www.energy.gov/oe/activities/technology-development/grid-modernization-and-smart-grid
} 


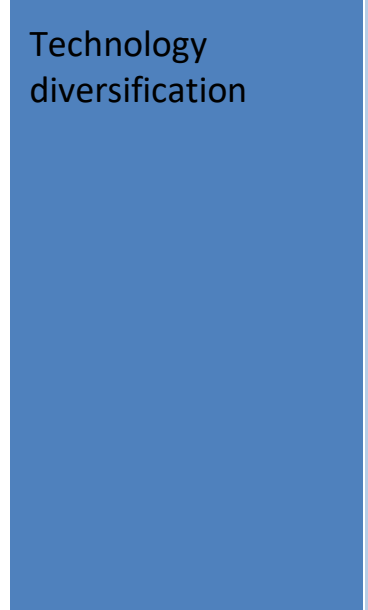

Lack of behind-themeter visibility

Performance issues
The rapid growth of new and innovative technologies across all domains of the evolving grid has led the expanding universe of technology developers and vendors to employ diverse standards and protocols for digitized communications and/or control. This diversity complicates the communications required to accurately measure, analyze, predict, protect, and control the grid of the future. Achieving the envisioned smart grid benefits, including increased efficiency, reliability, affordability, and renewables use, requires robust communications.

The lack of a common language enabling accurate communications and coordination among devices and systems is at the root of the current interoperability challenge. The lack of common requirements for interoperability can raise costs, slow device development, and impede integration/optimization.

In the smart grid, the distribution domain will have increased sensing and control capabilities and will communicate in a more granular fashion with the operations domain in real-time to manage the complex power flows associated with new technologies, a more dynamic markets domain, and other environmental and security-based factors. This dynamic indicates a need for improving distribution system observability and awareness.

The proliferation of new devices and equipment is particularly pronounced on the end-user side. Private and commercial consumers now have access to an unprecedented and growing array of devices for managing, generating, and storing energy in the home, vehicle, or building. Distribution utilities need a way to see the amount of energy storage on these behind-the-meter (BTM) devices. The issues go beyond a common language for communicating: questions arise over data ownership, privacy, and potential control over resources under conditions to be specified.

Lack of a clear consensus on interoperability standards currently impedes market acceptance of devices and adds to costs. At a more granular level, utility operators do not know the precise internal functions of any particular device or piece of equipment - the classic "black box" enigma. Performance claims may be clear, but the details of operation or critical algorithms (typically proprietary) are not disclosed-leaving open the question of whether a piece of equipment will perform as advertised once connected to a specific system.

Utilities are keenly aware of the unique characteristics of their own system, and they are skeptical that an off-the-shelf product will necessarily deliver the required performance under the unique circumstances of their system. As a result, vendors (particularly small or startup enterprises) must often prove a product's compatibility and performance for each utility, costing both the utility and vendor considerable time and money.

In some cases, this hurdle leads utilities to stay with a particular vendor once its equipment has been proven to work for them. While vendors may appreciate such customer loyalty, this caution has the potential to substantially slow utility adoption of improved equipment options and pose a significant market hurdle to technology innovators. 


\section{Fault tolerance in} inverters
The role of inverters on the distribution grid has gained significant focus with the increased integration of renewable energy sources, energy storage, and non-frequency responsive loads. ${ }^{13}$ Individually, inverters may protect themselves well when the grid experiences a fault, but they do not collectively help mitigate faults by maintaining system inertia or proactively interrupting cascading grid faults.

Inverter manufacturers may offer proprietary control for fault mitigation, however that diversity is not suited to ensure grid scale fault response. Unless a standard were developed vendors are unlikely to add the needed advanced inverter capabilities because of the associated costs. Methods are needed to test for and determine standard fault contributions for different types of inverters; understanding these impacts would help protect microgrids and aid in power electronics development. A related need is to identify adaptive smart inverter set-ins. The testbed could help to identify what those IEEE Std. 1547 set-ins are in aggregate at small scale.

\section{Access}

All stakeholders with an interest in interoperability face significant challenges in gaining access to or knowledge about the variety of existing smart grid testbeds; real-world, high-quality utility data in consistent format; a variety of specialized expertise; and stronger collaboration vehicles.

\section{Scarce information} on testbeds
Many stakeholders who might derive significant benefit from working with a particular type of testbed have limited awareness of the different types of existing smart grid testbeds, their roles and purposes, specialized capabilities, outputs, partnering opportunities, terms, and locations. Some vendors might be interested in working with testbeds in ways that could reduce the need to prove their products to each unique utility. Large utilities may be interested in working with testbeds to solve a wide range of emerging issues. Smaller utilities typically have neither funding for research nor testing facilities, and they are often too small and unique to influence technology developers. The immediate customer service orientation of many utilities also means that utility staff are often too busy to take advantage of a testbed, even if they had access.

\footnotetext{
${ }^{13}$ www.energy.gov/sites/prod/files/2019/02/f59/Smart\%20Grid\%20System\%20Report\%20November\%202018 1. pdf
} 


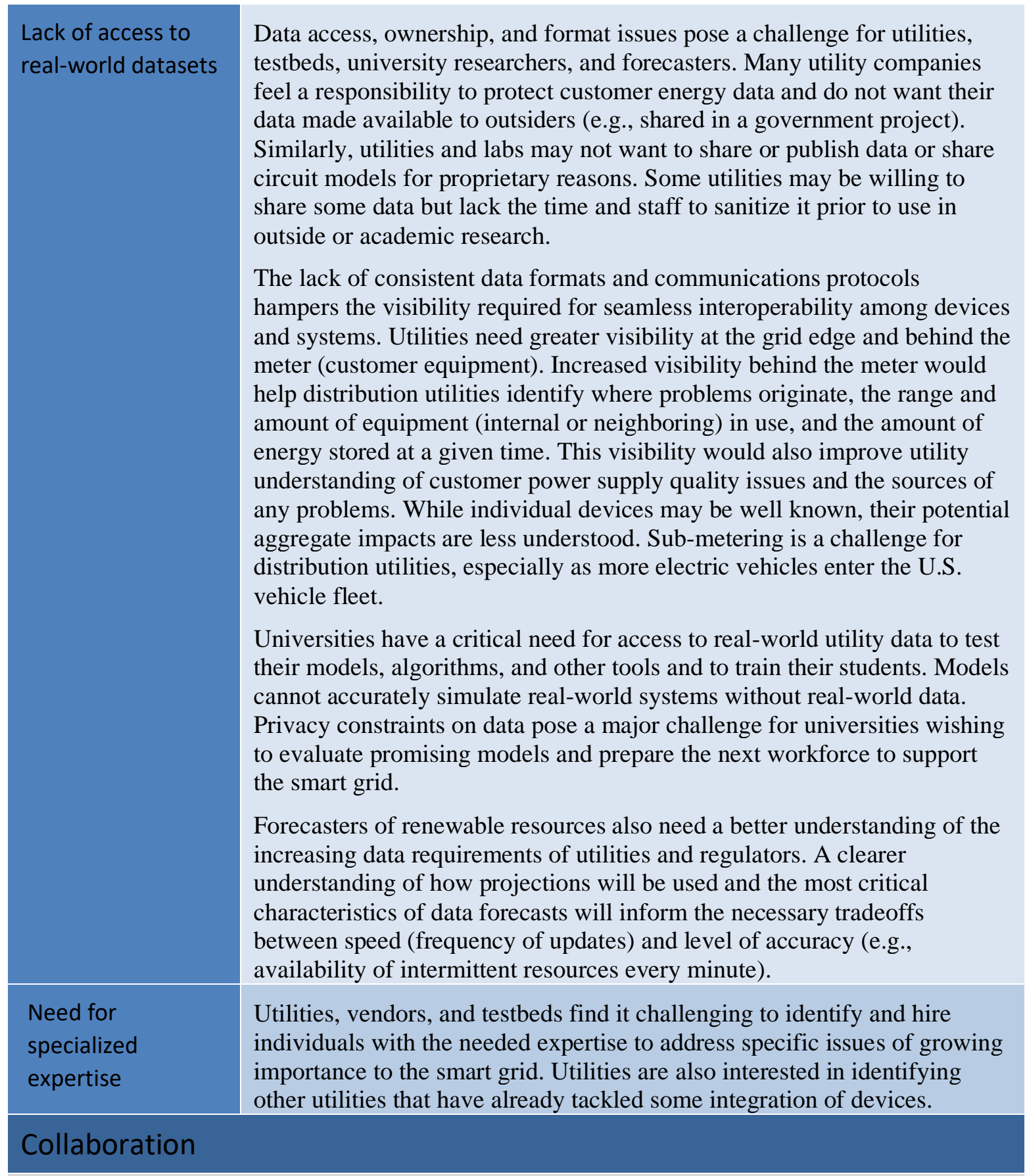

To enable broader collaboration among testbeds and accelerate progress toward smart grid interoperability, the workshop participants identified several challenges to be addressed.

Lack of shared communications platforms/language
The number, variety, capabilities, and range of devices are growing at an unprecedented rate. Testbeds need better ways to encourage and incentivize collaboration with a range of stakeholders to help make sure these devices communicate at the required speed and accuracy. 


\begin{tabular}{l|l}
$\begin{array}{l}\text { Competing goals } \\
\text { and cultures }\end{array}$ & $\begin{array}{l}\text { Utilities, vendors, universities, and government have different priorities, } \\
\text { responsibilities, timeframes (e.g., industry moves more quickly than } \\
\text { academia or government), and means of financial support that can pose } \\
\text { hurdles to collaboration. Protection of data and intellectual property can } \\
\text { also raise issues of trust and competitiveness. } \\
\text { Utilities may be more likely to work with big-name universities or } \\
\text { researchers, but smaller schools and programs also offer top-notch } \\
\text { research opportunities. }\end{array}$ \\
\hline $\begin{array}{l}\text { Intellectual } \\
\text { property (IP) } \\
\text { concerns }\end{array}$ & $\begin{array}{l}\text { ownership protect original IP as well as any new IP produced through } \\
\text { collaboration with others. Collaborative efforts cannot be successful in the } \\
\text { absence of trust, so all potential cases of IP protection or assignment need } \\
\text { to be explored and agreed upon before prior to collaborative research. }\end{array}$
\end{tabular}

\section{Opportunities}

Testbeds of various types have diverse opportunities to support progress toward interoperability and other smart grid goals. Key opportunities lie in the expansion of testbed capabilities and collaborative efforts.

\section{Capabilities}

Testbeds can strengthen their contributions by bolstering their in-house expertise and testing/validation capabilities, by improving their data and modeling to explore tradeoffs and enhance visibility behind the meter, and by clarifying the assumptions and requirements to enable universal applications.

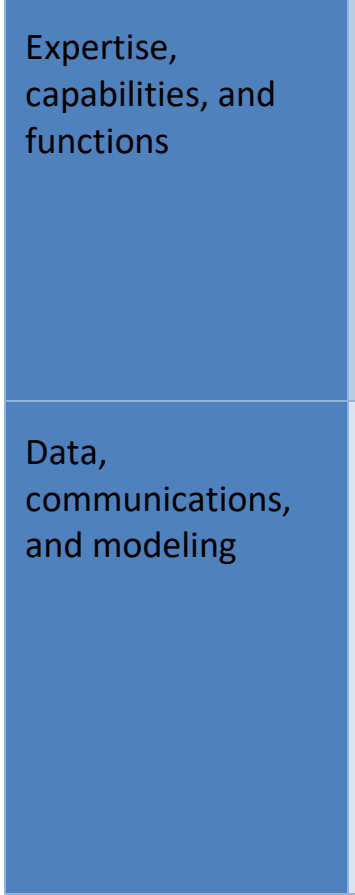

By incorporating a variety of highly specialized utility engineering expertise, testbeds could become better equipped to investigate models within the context of a utility environment. Use of real-world datasets will enable testbeds to validate simulation models and assess the reliability (scope, accuracy, and timing) of critical interfaces between devices and equipment. Potentially, industry partners or associations could be granted remote access to testbed hardware in the loop to expedite problem solving in real time.

Testbeds have an opportunity to work with utilities to provide good quality, sanitized datasets to labs, universities, and others who need to validate or calibrate their own models and computational tools. In addition, testbeds should explore the potential tradeoffs in setting up a testbed that is highly flexible and adaptable to emulate broadly diverse scenarios versus one that is highly accurate at modeling a specific or narrower range of scenarios.

More accurate utility data will help to create more accurate lab scenarios, but testbeds could also help clarify situations in which a high level of accuracy is needed at the device level or only at the fleet level. Moreover, in the absence of an ANSI metering standard for DC circuits, testbeds can 


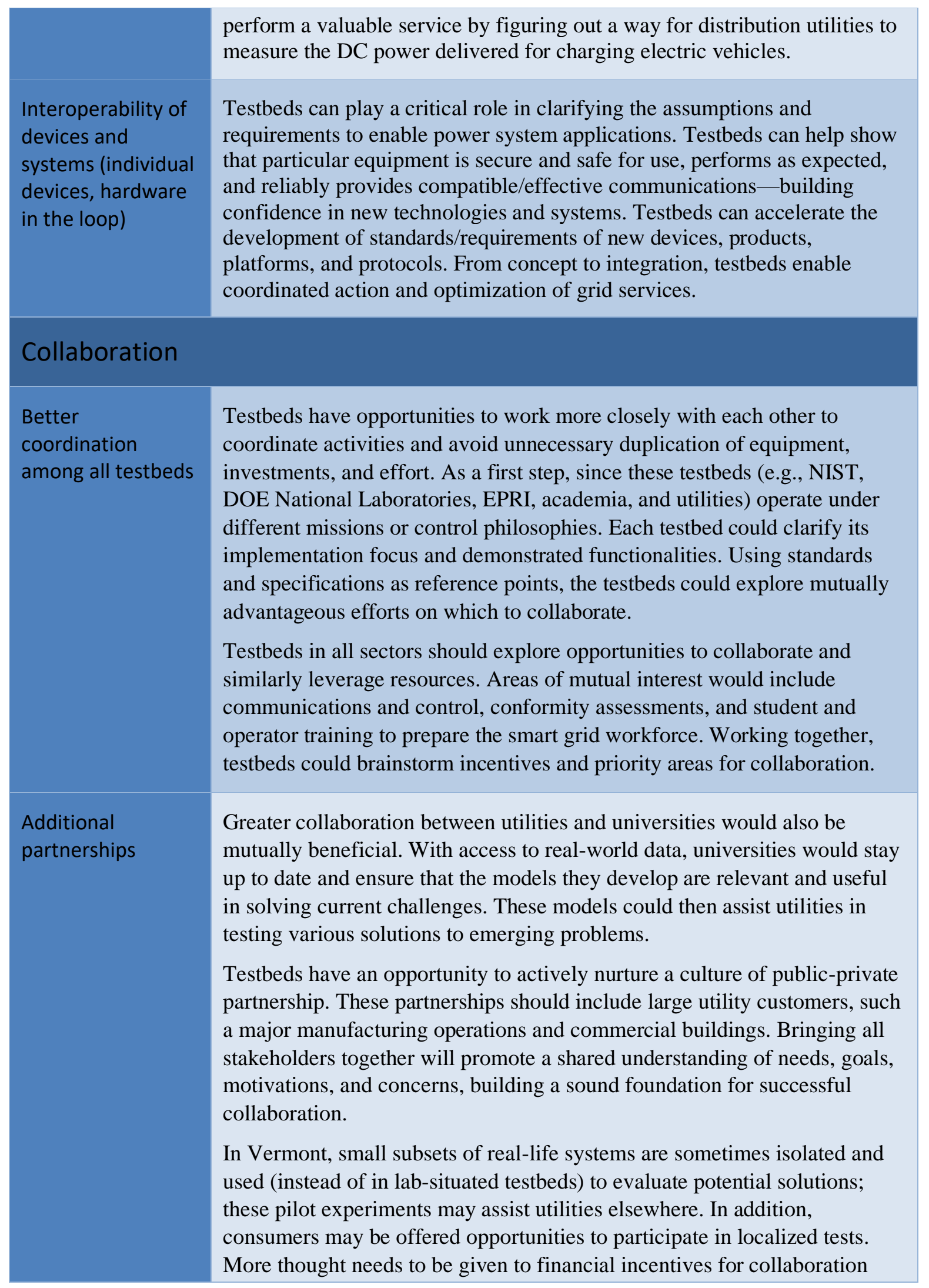


including developing grant programs to build and sustain the human capital and technical expertise required to run a collaborative testbed program.

\begin{tabular}{|c|c|}
\hline & Gaps \\
\hline \multicolumn{2}{|l|}{ Technical } \\
\hline $\begin{array}{l}\text { Interpretation } \\
\text { of novel } \\
\text { metrics }\end{array}$ & $\begin{array}{l}\text { The rapidly evolving nature of smart grid devices and functions raises several } \\
\text { technical issues that have not yet been addressed by testbeds. For example, } \\
\text { some utilities have expressed alarm upon obtaining system measurements that } \\
\text { had not been previously available. New and surprising observations that emerge } \\
\text { may require time for study and interpretation; they may reflect long-standing } \\
\text { conditions that had not been apparent in the absence of measurement } \\
\text { capabilities. }\end{array}$ \\
\hline $\begin{array}{l}\text { Circuit } \\
\text { capacity and } \\
\text { control theory }\end{array}$ & $\begin{array}{l}\text { Questions have arisen around the continuing validity of established ANSI } \\
\text { standards under conditions on the new grid. For example, what is the hosting } \\
\text { capacity of a circuit? How can we find out or measure whether control limits are } \\
\text { changing? Similarly, new and projected requirements for information exchange } \\
\text { on the grid need to be categorized and cataloged as a first step toward } \\
\text { developing robust and effective control algorithms. }\end{array}$ \\
\hline \multicolumn{2}{|l|}{ Data } \\
\hline $\begin{array}{l}\text { Reference } \\
\text { datasets }\end{array}$ & $\begin{array}{l}\text { Universities and other model developers critically need access to utility data so } \\
\text { that the models they develop accurately reflect grid operations in the real world. } \\
\text { Given this data, they would then be able to run low-cost models to simulate and } \\
\text { explore grid functions (e.g., fault detection). }\end{array}$ \\
\hline $\begin{array}{l}\text { Data } \\
\text { interfaces }\end{array}$ & $\begin{array}{l}\text { Data interfaces pose a major challenge, even with mostly open source software. } \\
\text { The industry needs a model for data exchange and data streaming. Broadly, how } \\
\text { does a testbed represent a model and exchange it with another testbed (e.g., } \\
\text { Modelica, XML)? Currently, an intense manual effort is required to share an } \\
\text { inquiry or investigation with another testbed. }\end{array}$ \\
\hline \multicolumn{2}{|l|}{ Scalability } \\
\hline Emulation gap & $\begin{array}{l}\text { Testbed should employ similar assumptions about aggregation and scalability to } \\
\text { better enable comparability across testbeds. There needs to be standardization in } \\
\text { the terminology and definitions used to communicate assumptions made about } \\
\text { scalability and uncertainty. Guidance and best practices on how to self-assess the } \\
\text { limits of emulation would be beneficial. }\end{array}$ \\
\hline \multicolumn{2}{|l|}{ Behavior } \\
\hline $\begin{array}{l}\text { Human } \\
\text { factors }\end{array}$ & $\begin{array}{l}\text { In the complex smart grid environment, users are empowered to act on real-time } \\
\text { information on energy and price; consumers interact with smart HVAC, EV }\end{array}$ \\
\hline
\end{tabular}




charging, and whole building technologies. To avoid future problems, testbeds
must accurately model people and their decision-making processes.
For example, it cannot be assumed that everyone will be on board with a specific
load shedding program. What is a realistic level of expected participation, and
what factors might change those levels? How can testbeds simulate the effects of
human behavior?

\section{Potential Roles of Collaborative Testbeds in Achieving Goals of Smart Grid Stakeholders}

Collaboration accelerates innovation, and it is generally best to involve all stakeholders as early as possible in the process of developing solutions. Prior to identifying the potential roles of testbeds in helping achieve the smart grid goals of diverse stakeholders, participants at the Tennessee workshop decided to first identify and understand the main testbed business models or use cases. Through open discussions, they identified the first six use cases shown in the box on the right. Workshop participants in Vermont later reviewed these six cases and added a seventh, underscoring the great value in learning from failures and

\section{Proposed Key Testbed Use Cases}

1. Training (e.g., operators/students)

2. Federal grant-based technology development (e.g., demonstrate first-of-its-kind technology)

3. User facilities (user facilities at national labs work with industry via diverse agreements)

4. General science or basic research

5. Conformity assessment/validation

6. Patents/IP (proprietary development)

7. Demonstration/Post-mortem analysis abandoned pathways.

\section{Testbed Goals}

The list of distinct business models can potentially help motivate or incentivize cross-domain collaborations. Participants suggested that a subsequent effort to map relationships among these use cases (e.g., sequential, parallel, and/or feedback loops) could help define the most promising potential 
pathways for testbed collaborations. For example, analysis may determine whether collaborations are likely to be most effective among same use cases or across two or more distinct cases (possibly by type).

Collaborations across testbeds that engage a broad range of stakeholders have the potential to address important questions on the following topics:

- Harmonics on the distribution grid (including transition to digital generation and digital loads);

- Interfaces between smart buildings or microgrids and the distribution grid;

- Non-volumetric metering and services (e.g., monitoring and control), including data ownership, privacy, and trustworthiness of non-physical meters;

- Cybersecurity risks and benefits and the exploration of new management options (e.g., every device may not require ultra-high protection, but a better understanding is required of the tradeoffs involved);

- Accuracy tradeoffs between device-level and fleet-level awareness and control; and

- Control theory for distributed energy resources.

\section{Testbed Collaborations to Serve Stakeholder Goals}

\section{Utility Goals}

As regulated entities, utilities are inherently risk averse. The changing grid environment is forcing many utilities out of their comfort zone and into new territory fraught with uncertainties. Effective collaborations across testbeds could help technology developers increase product flexibility and widen applications to address critical utility concerns about technology safety and reliability.

Common

testbed

protocols

of data and

testing

opportunities
Common testbed protocols or requirements could give utilities and other stakeholders greater confidence in equipment and device performance. Proven performance across diverse testbeds could increase market acceptance of emerging technologies that improve grid operations. Utilities that interact with testbeds are also more likely to have their concerns heard and to become better informed about solutions.

The greatest challenge facing utilities is the need to optimize interactions across all components to keep customers happy and make the overall system as efficient, affordable, reliable, and sustainable as possible. This challenge continues to grow as the scope and diversity of interconnected systems continues to expand. Development of standards for testing grid devices and equipment (including control strategies) across manufacturers/devices will lead to more robust and flexible products, which is an important step toward the ultimate goal of interoperability (seamless communications and optimized control across systems).

Testbeds can provide training and hands-on experience for real-world end users, including utility staff responsible for the operations and maintenance of different types of equipment. 


\begin{tabular}{|c|c|}
\hline $\begin{array}{l}\text { Testbed white } \\
\text { papers and use } \\
\text { cases }\end{array}$ & $\begin{array}{l}\text { White papers can help justify further penetration of renewables or help solve } \\
\text { problems at utilities. For example, past white papers published by NIST and the } \\
\text { National Laboratories provide supporting evidence for solutions to system } \\
\text { performance problems. By soliciting utility input on scope prior to paper } \\
\text { development, NIST could expand the usefulness of these papers in helping } \\
\text { utilities solve real-world problems. }\end{array}$ \\
\hline \multicolumn{2}{|l|}{ Vendor Goals } \\
\hline $\begin{array}{l}\text { Utility } \\
\text { confidence in } \\
\text { new } \\
\text { equipment }\end{array}$ & $\begin{array}{l}\text { Testbeds could potentially help build utility confidence in new equipment, } \\
\text { reducing the time and money that vendors now spend proving their equipment to } \\
\text { each utility individually. Standard simulations for product testing could be useful } \\
\text { but may not meet the needs in all test cases. Greater utility confidence (justified) } \\
\text { in novel products could help expedite grid modernization. }\end{array}$ \\
\hline $\begin{array}{l}\text { Understanding } \\
\text { emerging } \\
\text { utility needs }\end{array}$ & $\begin{array}{l}\text { Most individual utilities are not large enough to influence international vendors, } \\
\text { yet vendors are interested in hearing what utilities want and obtaining greater } \\
\text { clarity on their assumptions. Testbeds could potentially assist vendors by } \\
\text { identifying common needs across utilities. }\end{array}$ \\
\hline \multicolumn{2}{|c|}{ University Goals } \\
\hline $\begin{array}{l}\text { Access to real- } \\
\text { world data }\end{array}$ & $\begin{array}{l}\text { Universities, other academic institutions, and testbeds all need access to real- } \\
\text { world utility models, data and use cases; testbeds may be able to collaborate with } \\
\text { utilities and universities to improve this access. Data sharing can drive } \\
\text { innovation but must allay privacy concerns. }\end{array}$ \\
\hline $\begin{array}{l}\text { Technical } \\
\text { knowledge and } \\
\text { innovation }\end{array}$ & $\begin{array}{l}\text { Collaborative testbeds can solve technical issues for certain stakeholders and } \\
\text { share generalized outcomes, e.g., show how different technologies in a microgrid } \\
\text { can impact the end goal. }\end{array}$ \\
\hline $\begin{array}{l}\text { Student } \\
\text { training }\end{array}$ & $\begin{array}{l}\text { Testbed collaborations can also foster industry-academia integration and real- } \\
\text { world training for students. Internships educate students about system operations } \\
\text { and data needs for real-world simulations, equipping them for the future smart } \\
\text { grid workforce. }\end{array}$ \\
\hline
\end{tabular}

\section{Ideal Components/Elements for Successful Testbed Collaboration}

Collaboration across testbeds may be the most effective strategy to feed the innovation cycle and relate the results to requirements development acceptable to all stakeholders. Suggestions on the critical ingredients for successful testbed collaborations cluster under the broad categories of coordination/trust and facilitated sharing. Workshop participants further suggested topics that would benefit from attention from testbed collaborations. 


\section{Ideal Elements for Testbed Collaboration}

\section{Trust \& Coordination}

Mechanisms

to promote

trust

Interdiscipli

nary

approach

Real-world

relevance
A trust ecosystem will promote true collaboration. Establish trusted mechanisms for sharing data, permissions, transfers, etc.

A broad range of experts in relevant areas is needed. Successful collaborations will need protection experts, computer science researchers, control theorists, signal processing experts, communications experts, experts on cyber-physical simulations, utility engineers and planners, major end users, vendors, and manufacturers.

Testbeds should represent the real world. Focus on current, tangible needs by partnering with utilities. Testbed products should be transferable to utilities/manufacturers.

\section{Sharing}

\section{Shared objectives}

Central clearing house \& information exchange

Successful collaborations need mutual benefits, financial incentives, strong objectives, trust, and streamlined processes to protect privacy and enable safe sharing.

A central database to serve as a repository and pipeline for data and coordination is needed. A repository of contact information for who to turn to for help with specific questions. The software community can provide open source software. A formal sharing platform should protect privacy. Testbed roles should be highlighted:

Patent/IP testbeds could feed information to vendors or SDOs.

General Science testbeds could share information on requirements that could help standardize interfaces among devices.

User groups can share templates and guidelines.

Utilities could share information with labs and universities to support training and progress.

Federal testbeds: software open source, available tools, data, info exchange. Learn from military micro grids.

\section{Focus Areas for Successful Testbed Collaboration}

Testbed

flexibility vs

realism

Cyber-

physical

security
Two paradigms can often be at odds with each other. Compromise must be careful and deliberate. Successful aggregation and scalability require finding the right balance among tradeoffs. Are we all making the same assumptions? Can results be replicated?

Cyber-physical security issues are always a concern - and growing. Internet-based devices also create a need for better training in best cybersecurity practices. 


\begin{tabular}{|c|c|}
\hline $\begin{array}{l}\text { Sensor } \\
\text { ubiquity vs. } \\
\text { fidelity }\end{array}$ & $\begin{array}{l}\text { How many system measurements are needed to obtain an adequate understanding of } \\
\text { the system? Assess the appropriate sensor penetration levels needed for different } \\
\text { levels of accuracy. Assess the impacts of using a smattering of lower accuracy } \\
\text { sensors vs a few highly accurate sensors in strategic places. How does the chosen } \\
\text { sensing approach affect testbeds? What is the sensor accuracy for existing testbeds? } \\
\text { What should it be? }\end{array}$ \\
\hline $\begin{array}{l}\text { Explore data } \\
\text { access rights }\end{array}$ & $\begin{array}{l}\text { Does a utility have the right to see the energy data of individual residences and that } \\
\text { of other market platforms? Is the right conditional? Who decides? Can utilities } \\
\text { share their models and data with testbeds? What is the risk if the data were } \\
\text { disclosed? What level of trust is needed between testbeds and utilities, how can the } \\
\text { utility models and data be secured? }\end{array}$ \\
\hline Time scale & $\begin{array}{l}\text { Data sharing in testbeds should mimic real-world situations. Make sure testbeds are } \\
\text { quick enough to consider real dynamics, e.g., many do not update control loops at } \\
\text { synchrophasor speeds. }\end{array}$ \\
\hline $\begin{array}{l}\text { Uncertainty } \\
\text { propagation }\end{array}$ & Improve understanding of the impacts of uncertainties across systems \\
\hline $\begin{array}{l}\text { Enhanced } \\
\text { visualization }\end{array}$ & $\begin{array}{l}\text { Improve utility capacity to look at power quality behind the meter and aggregated } \\
\text { device impacts at the grid edge. }\end{array}$ \\
\hline $\begin{array}{l}\text { Widely } \\
\text { applicable } \\
\text { models }\end{array}$ & $\begin{array}{l}\text { Continual validation and adjustment of models as they are applied to more real- } \\
\text { world systems move the models toward wider applicability/validity. }\end{array}$ \\
\hline $\begin{array}{l}\text { Lower utility } \\
\text { risk }\end{array}$ & $\begin{array}{l}\text { Demonstrating device performance and mitigating risk can improve the comfort } \\
\text { level of many utilities with an asymmetric risk profile (improvements vs. outages). }\end{array}$ \\
\hline $\begin{array}{l}\text { Understandi } \\
\text { ng model } \\
\text { validity } \\
\text { amidst } \\
\text { change }\end{array}$ & $\begin{array}{l}\text { How do you know that your testbed is valid? Need ways to help people validate } \\
\text { what they have-even for the grid of the far future with the potential of very high } \\
\text { penetration of renewables. }\end{array}$ \\
\hline
\end{tabular}




\section{NeXt Steps}

This summary of the two workshops on smart grid testbeds and collaborations is intended to motivate and guide the following next steps:

1. Build a coordinated smart grid testbed agenda for industry, government, and academia. Pay particular heed to innovations emerging from startup enterprises, research organizations and smaller technology vendors who rely on access to shared testbed resources to validate and communicate the effectiveness of their solutions. Larger utilities, standards organizations and government regulators also stand to benefit from better coordination and access to testbed resources.

2. Identify and remediate strategic gaps in smart grid efforts that could impede progress. Particularly relating to testbeds; gaps include may include technologies that are currently underrepresented, stakeholders with insufficient access to testing, and regulators or policy makers who do not have visibility into the technology maturation process to make proactive strategic decisions.

3. Provide an understanding of the important role of testbeds in the development and deployment of smart grid technologies and systems.

4. Guide future research programs in the smart grid area at NIST and the provide feedback for the expansion of its Smart Grid Testbed Facility.

5. Assist with developing testing and standardization protocols not just for the smart grid technologies of today but also consider the evolution of grid governance which may include many more stakeholders such as system integrators and operators offering niche grid services and operators of distributed energy resources driving innovation in grid operations. 


\section{APPENDIX A: PARTICIPANT LISTS}

\section{Workshop at University of Tennessee, Knoxville TN, April 11, 2019}

Lisa Beard, University of Tennessee, Knoxville

Paul Boynton, National Institute of Standards and Technology

Hantao Cui, University of Tennessee, Knoxville

Samuel DeLay, Tennessee Valley Authority

Corey Dickens, National Institute of Standards and Technology

Vaibhav Donde, Lawrence Livermore National Laboratory

Ben Ealey, Electric Power Research Institute (EPRI)

Norma Goldstein, Association of Community College Trustees (ACCT)

Allen Goldstein, National Institute of Standards and Technology

Avi Gopstein, National Institute of Standards and Technology

Fran Li, University of Tennessee, Knoxville

Yilu Liu, University of Tennessee, Knoxville, and Oak Ridge National Laboratory

Ben Ollis, Oak Ridge National Laboratory

Dwight Rizy, Oak Ridge National Laboratory

Aaron Snyder, EnerNex

Fatima Taousser, University of Tennessee, Knoxville

Emmanuel Taylor, Energetics

Leon Tolbert, University of Tennessee, Knoxville

Kevin Tomsovic, University of Tennessee, Knoxville

Fred Wang, CURENT, University of Tennessee, Knoxville

Jingxin Wang, University of Tennessee, Knoxville 


\section{Workshop at University of Vermont, Burlington VT, April 23, 2019}

Mads Almassalkhi, University of Vermont

Paul Boynton, National Institute of Standards and Technology

Joshua Burroughs, Vermont Electric Power Company

Morgan Casella, Dynamic Organics

Paget Donnelly, Energetics

Luis Duffaut Espinosa, University of Vermont

Andrew Elliston, Burlington Electric Department

Bernadette Fernandes, Vermont Electric Power Company (VELCO)

Stephen Fitzhugh, Norwich University

Daniel Fredman, Vermont Energy Investment Corporation (VEIC)

Allen Goldstein, National Institute of Standards and Technology

Avi Gopstein, National Institute of Standards and Technology

Robert Horton, Green Mountain Power (GMP)

Munir Kasti, Burlington Electric Department

Adil Khurram, University of Vermont

Daniel Kirk-Davidoff, UL

Tom Linn, LinnWrite

Steffi Muhanji, Thayer School of Engineering at Dartmouth

Simon Odi, Comm-Edison

Hamid Ossareh, University of Vermont

Thomas Prevost, Weidmann Electrical Technology Inc

Christopher Root, Vermont Electric Power Company (VELCO)

Allison Ross, Vermont Energy Investment Corporation (VEIC)

Peter Rossi, Vermont Electric Cooperative, Inc.

Douglas Savino, Orange \& Rockland Utilities

Enis Sehovic, City of Burlington

Ori Shmul, Orange and Rockland Utilities

Steve Thompson, Smarter Grid Solutions

Graham Turk, Green Mountain Power

Bradley Williams, Burlington Electric Department

Tucker Williams, Vermont Electric Cooperative

Alexa Woodward, UVM Foundation

Steve Young, UL 


\section{ApPendix B: WoRKShop(s) AgENDA}

\begin{tabular}{|c|c|}
\hline \multicolumn{2}{|r|}{ NIST Workshop on Smart Grid Testbeds and Collaborations } \\
\hline 9:00 AM & Welcome \\
\hline 9:30 AM & NIST Perspective on Testbed Collaboration-Paul \\
\hline 9:40 AM & NIST Smart Grid Testbed-Allen, Avi \\
\hline 10:00 AM & Break \\
\hline 10:15 AM & $\begin{array}{l}\text { Breakout Session: Stakeholder Input on Collaborations } \\
\text { Questions: } \\
\text { 1. What do you see as current, major challenges, opportunities and gaps in Testbed } \\
\text { capabilities (e.g. technical, expertise, collaboration, accessibility)? } \\
\text { 2. Given your organizations/ goals for smart grid stakeholders, how can } \\
\text { Collaborative Testbeds help achieve these? } \\
\text { 3. What are the ideal components/elements for successful Testbed collaboration? }\end{array}$ \\
\hline 11:45 AM & Lunch \\
\hline 1:00 PM & Stakeholder Panel: Experiences with Testbed Collaborations--Paul \\
\hline 2:15 PM & Break \\
\hline 2:30 PM & Report Outs and Next Steps \\
\hline 4:00 PM & Tour of Facility \\
\hline
\end{tabular}




\title{
APPENDIX C: ACRONYMS
}

\author{
CPS Cyber-Physical Systems \\ DER Distributed Energy Resource \\ EISA Energy Independence and Security Act of 2007 \\ EMS Energy Management System \\ EPRI Electric Power Research Institute \\ EV Electric vehicle \\ FERC Federal Energy Regulatory Commissions \\ GMLC Grid Modernization Lab Consortium \\ IEEE Institute of Electrical and Electronics Engineers \\ IO Interoperability \\ IoT Internet of Things \\ IIoT Industrial Internet of Things \\ IP Intellectual property \\ ISO International Standards Organization \\ NEISO New England ISO \\ NIST National Institute of Standards and Technology \\ NSF National Science Foundation \\ PMU Phasor measurement unit or power measurement unit \\ PNNL Pacific Northwest National Laboratory \\ PSC Public Service Commission \\ PUC Public Utility Commission \\ RFP Request for proposal \\ SDO Standards Development Organization \\ SEPA Smart Electric Power Alliance \\ SCADA Supervisory Control and Data Acquisition \\ SG Smart Grid \\ UTC Coordinated Universal Time \\ UTK University of Tennessee, Knoxville \\ UVM University of Vermont \\ VEIC Vermont Energy Investment Corporation \\ VOLTTRON Open-source and secure execution and communications platform from PNNL \\ WECC Western Electricity Coordinating Council
}




\section{Addendum: StakehOlder PERSPECTIVES AS RECORDED DURING BREAKOUT SESSIONS}

\section{Gaps}

- Standards

- More standards are needed in certain areas

- Many standards that exist have few actual requirements

- Many requirements are unmeasurable (ambiguity)

- Lack of compliance testing programs

- Users groups

- Few groups look at the big picture (no testbed focus)

- Survey of testbeds/capabilities (one for National Labs only)

- "Common knowledge" of testbed-related topics (from bringing testbeds together)

- Guidelines and best practices

- Common testing scenarios (e.g., forced oscillation scenario with signals to enable comparison of results across testbeds)

- Few exist (testbeds and vendors do their own thing)

- No standard testing

- No standard events to test against

- Validated against real world (emphasized need for validation)

- Toolsets

- Need open source tools (many are proprietary)

- Training materials (including for open source)

- Documentation

- Tutorials

- Examples

- Data interfaces to enable testbed collaboration

- Common formats (some exist but not widely adopted)

- Data exchange model

- Sharing data is labor intensive

- Uncertain requirements

- Emulation gap (inability to verify that models reflect real world; data issues and use cases)

- Aggregation and scalability

- Similar assumptions among testbeds

- Steady state vs actual dynamics of the grid and emerging grid

- Technology Roadmap

- Discrete tasks

- Roles

- Trigger points (choices)

- Milestones

- Collective vision

- Accounting for human behavior

- Simulate the effects of user behavior

- Consumer interacts with technologies

- Model decision making

- Acceptance of load shedding programs 


\section{Challenges}

- Data Sharing

- Much of the real-world data:

- Proprietary to utilities

- Considered security risk

- Needs NDA/CRADA to share

- Must be "sanitized" before release (not their job)

- Owners don't have time, funding, motivation

- Need a trusted, secure repository

- Many formats exist

- Few standards (often unique)

- Most of the data does not comply

- Access to Testbeds

- Lack of knowledge (more utilities and vendors in VT)

- What is a testbed?

- What do they offer? (Do IEEE bus models used in many testbeds reflect real world?)

- Where are the existing testbeds?

- Is there a list of them and their capabilities?

- How do people get involved in the work going on in the testbeds?

- Utilities each operate unique systems

- How do they influence technology developers?

- No funding for testbeds of their own

- Busy on other issues

- Access to expertise

- Hard to find the right SME

- Hard to find people with field experience

- Technical

- Rapid change

- Models must be validated

- Proprietary devices (e.g., wind turbines)

- No models or poor models exist

- Every manufacture has different control loops.

- Differing fault behaviors for inverters.

- No standard fault profiles for testing

- No models, protection and control schemes for aggregated inverters

- No behind-the-meter visibility.

- BTM storage

- Distributed generation

- Controllable loads 


\section{Opportunities in Testbed Collaboration}

- Build confidence in new technologies and systems

- Equipment performs as expected: Secure, safe, and interoperable

- Identify gaps in data, communications, and modeling (we don't even know what we don't know)

- Provide data needed for models and computational tools

- Use real-life systems as testbeds

- Pilots on the grid

- Limit impacts for safety

- Clarify data tradeoffs

- Flexible testbed setup vs modeling accurate scenarios

- Data creates accurate lab scenarios

- Understanding scalability

- Validate interoperability of devices and systems

- Individual devices

- Hardware in the loop

- Clarify assumptions

- Devices constantly changing

- Consumers can participate

- $\quad$ Requirements enable universal applications

- Expand collaboration

- Increase industry-university partnership; industry moves faster than academia; academia needs to stay relevant

- Overcome financial and trust issues

- Nurture public-private partnership

- Expanding testbed capabilities

- Variety of expertise

- Investigate models.

- Incorporate datasets/model interfaces.

- Remote use of hardware?

- Real-time, hardware in the loop?

- Better coordinate among all testbeds

- Testbeds can collaborate on training

- Conformity assessment (see where needed)

- Incentivize.

- Collaboration among testbeds

- Avoids duplications

- Show reference implementations

- Demonstrates functionality under different control philosophies

- Include large utility customers

- Outreach

- Engagement

- Discover utility needs

- Accelerate standards development (collaborative process involving testbeds)

- Platforms

- Protocols

- Devices

- Functions

- Lack of standards for interoperability impedes market acceptance of devices and adds to costs. 


\section{Achieving Goals via Collaboration}

- Business models for testbeds (partner with type that is different from you)

- Training (train utility operators for grid-edge control/future, university students)

- Federal grant-based technology development (unique)

- User facilities (National Lab model)

- General science (fundamental science, \$ secure)

- Conformity assessment/validation

- Patents/IP (proprietary research by private companies)

- Demonstration/Post-mortems

- Common requirements or protocols

- Stakeholder confidence

- Improve market acceptance

- Standardization of data and testing

- Development of standards

- Common testing across manufacturers

- Common testing across utilities

- Technical knowledge and innovation

- Data sharing can drive innovation but must allay privacy concerns.

- Solve technical issues for stakeholders

- Generalize outcomes

- Show how different technologies can impact the end goal.

- Collaboration accelerates innovation

- Best to involve stakeholders early.

- Researchers

- Extends accessibility (to testbeds and each other)

- Coordination of goals and efforts (delegation)

- Peer review

- Academia

- Access to real-world use cases (utilities and government)

- Foster industry-academia integration

- Real-world training

- Internships (Labs)

- Vendors

- Reduce time and money proving equipment to each utility individually. (beyond conformity)

- Accelerate innovation and market entry.

- Utilities can communicate what they need (need to clarify assumptions)

- Utilities often stick with known vendors/products.

- Standard simulations for testing

- Standards

- Voluntary

- Consensus

- Measurable requirements

- Data driven

- Shared Use cases

- Justify further penetration of technology

- Help solve problems at utilities

- Supporting evidence for system performance and problems

- Focused research

- Testbed mandates and business cases; complementary roles; not all testbeds produce published results; private sector may choose not to publish 


\section{Ideal Components for Successful Testbed Collaboration}

- Mutual Benefit

- Complementary abilities

- Complementary equipment

- Complementary business models

- Financing

- Trust

- NDA / CRADA where needed

- Agreement of

- Private information

- Public information

- Secure repository

- Secure communications

- Communications

- Human to human

- Voice

- Email

- Human to machine

- Data transfer

- Passing of program, scripts

- Remote control, if needed

- Machine to machine

- Co-simulation if needed (remote)

- Expertise

- Interdisciplinary

- Operations

- Protection

- Electrical

- Electronics

- Signal processing

- Control theory

- Communications

- Cybersecurity, etc.

- Stakeholder engagement

- Sharing*

- Data (persistent issue, hard to solve)

- Models

- Scenarios

- Results

* open source where possible

- Validation

- Comparison to real-world responses (understand scalability)

- Models

- Scenarios

- Testbed results are transferrable to vendors and utilities 


\section{Use Cases for Testbeds}

- Understanding harmonics on neutral on distribution level due to DERs (data from sensors available: C. Root)

- 1547 requirements for distribution (ability to drive frequency back to nominal) withstand for momentary frequency changes

- Microgrids-Islanding (coupling/decoupling with distribution for grid following) toward smart inverters and DERMs

- Adjust smart inverters-Adaptive control for dynamic behavior for microgrids

- Understanding testbed scalability

- Interface of smart buildings and micro grids with the distribution grid

- Non-volumetric (metering other things than energy) metering and services, including trustworthiness of non-physical meters

- Cybersecurity: risk/cost vs. benefit

- Where is it really needed and not needed?

- Management options should be explored.

- Error/uncertainty propagation

- Sensor to Application

- Application to grid behavior

- Control theory for distributed energy resources

- Dynamic system performance 